\title{
GENERALIZED THOM SPECTRA AND TRANSVERSALITY FOR SPHERICAL FIBRATIONS ${ }^{1}$
}

\author{
BY NORMAN LEVITT
}

Communicated by W. Browder, February 27, 1970

1. A Poincaré duality space (abbreviated P.D. space) of dimension $n \geqq 0$ is a finite complex $M$ with the following property.

Let $M$ be embedded in $S^{n+k}, k$ large, and let $R$ be a regular neighborhood; then the inclusion $\partial R \subseteq R$, when converted to a fibration, has fiber a $(k-1)$-sphere.

Similarly a Poincaré cobordism $\left(W ; M_{0}, M_{1}\right)$ of dimension $n+1$ is a triad with the following property.

Let $W ; M_{0}, M_{1}$ be embedded in $S^{n+k} \times(I ;\{0\},\{1\})$ with relative regular neighborhood $R$ (i.e. $R \cap S^{n+k} \times\{i\}=Q_{i}$ is a regular neighborhood of $M_{i}$ in $\left.S^{n+k} \times\{i\}, i=0,1\right)$. Let $\bar{\partial} R=$ closure $\partial R-S^{n+k} \times\{0,1\}$. Then $\bar{\partial} R \subseteq R$ is a $(k-1)$-spherical fibration and $\bar{\partial} R \cap Q_{i}=\partial Q_{i} \subseteq Q_{i}$ is the induced $(k-1)$-spherical fibration.

A P.D. pair $M, \partial M$ is a P.D. cobordism $M ; \partial M, \varnothing$. If $W ; M_{0}, M_{1}$ is a P.D. cobordism then $M_{0}, M_{1}$ are P.D. spaces of one lower dimension. For a P.D. space $M$ let $\nu_{k}(M)$ be the fibration corresponding to $\partial R \subseteq R$; for a P.D. cobordism $W ; M_{0}, M_{1}$ let $\nu_{k}\left(W ; M_{0}, M_{1}\right)$ be the fibration corresponding to $\bar{\partial} R \subseteq R$.

A Generalized Thom Spectrum is a spectrum defined as follows: let $\xi_{k}: E_{k} \rightarrow B_{k}$ be a sequence of $(k-1)$-spherical fibrations, $k \geqq 1$. Let $\psi_{k}: B_{k} \rightarrow B_{k+1}$ be maps covered by spherical-fibration maps $\phi_{k}: \xi_{k} \oplus \epsilon \rightarrow \xi_{k+1}$.

Let the Thom complex $T\left(\xi^{x i}\right)$ be the space $\mathfrak{T}_{\xi_{k}} \cup c E_{k}$, i.e. the mapping cylinder of $\xi_{k}: E_{k} \rightarrow B_{k}$ union the cone on $E_{k}$ with the top of the mapping cylinder identified with the base of the cone. There are natural maps $\sum T\left(\xi_{k}\right) \rightarrow T\left(\xi_{k+1}\right)$. This forms the generalized Thom spectrum $T$.

Let $S$ be the spectrum got by taking $B_{k}=B_{k+1} \cdots=$ point; thus $S$ is the sphere spectrum. If $T$ is any spectrum as above, we assume that there are base points in each $B_{k}$, preserved by $\psi$. This gives an inclusion of spectra $S \subseteq T$.

A $T$-P.D. space (or simply $T$-space) is a P.D. space $M$ together with maps of spherical fibrations $f_{k}: \nu_{k}(M) \rightarrow \xi_{k}$ so that

AMS Subject Classifications. Primary 5568, 5710; Secondary 5550, 5730.

Key Words and Phrases. Thom complexes of spherical fibrations, Poincaré duality spaces, obstructions to transversality, Poincaré duality cobordism, surgery, Arf invariant, index, Top/PL, Pontrjagin-Thom construction.

${ }^{1}$ Partially supported by a Ford Foundation Grant. 


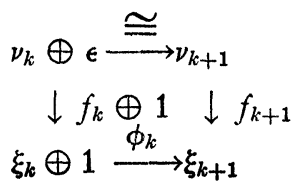

commutes ( $k$ large). We denote such an object by $(M, f)$.

Similarly, a $T$-cobordism is a P.D. cobordism $W ; M_{0}, M_{1}$ together with maps $g_{k}: \nu\left(W ; M_{0}, M_{1}\right) \rightarrow \xi_{k}$. Two $T$-spaces $\left(M_{0}, f^{0}\right),\left(M_{1}, f^{1}\right)$ are said to be $T$-cobordant iff there is a $T$-cobordism $W ; M_{0}, M_{1} ; g$ so that $f_{k}=g_{k} \circ i_{k}^{j}$, where $i_{k}^{j}$ is the natural map $\nu_{k}\left(M_{j}\right) \subseteq \nu_{k}\left(W ; M_{0}, M_{1}\right)$, $j=0,1$.

$T$-cobordism is an equivalence relation, and the operation of taking disjoint union defines a group on equivalence classes, so that we get a graded group $\Omega_{*}^{T}$ (graded by dimension). Of course, if we were dealing with PL or orthogonal bundles we would have the formula $\Omega_{*}^{T}=\pi_{*} T$, where $\pi_{*}$ denotes stable homotopy of the spectrum. However, it is well known that this is not the case for generalized Thom spectra. In particular, if $T$ arises from $\xi_{k}=$ universal orientable spherical fibration, i.e. $B_{k}=\mathrm{BSG}(k)$, then it is known that $\pi_{*} T$ is finite whereas $\Omega_{4 i}^{T}$ is of infinite order because there is a well-defined nontrivial homomorphism (the index) to the integers.

Our purpose then is to study the relationship between $\Omega_{*}^{T}$ and $\pi_{*} T$. In this paper, we state some important results. A full exposition will appear later.

2. The Pontrjagin-Thom construction yields a homomorphism $p: \Omega_{*}^{T} \rightarrow \pi_{*} T$. We will attempt to elucidate some properties of $p$. We restrict our attention to the case where $\xi_{k}$ is oriented and $\phi_{k}$ respects orientation.

We will first need to analyze a certain graded group $Q_{*}$, which is defined as the kernel of $p: \Omega_{*}^{S} \rightarrow \pi_{*} S$. It is easily seen that there are short exact sequences

$$
0 \rightarrow Q_{i} \rightarrow \Omega_{i}^{S} \stackrel{p}{\rightarrow} \pi_{i} S \rightarrow 0 .
$$

(The fact that $p$ is onto follows immediately from the fact that the isomorphism between ordinary framed cobordism of manifolds and $\pi_{*} S$ factors through $\Omega_{*}^{S}$.)

Lemma 1. For $i \geqq 5, Q_{i}=\pi_{i} G /$ Top. Recall $\pi_{i} G /$ Top $=0$, $i$ odd; $Z, i \equiv 0(4) ; Z_{2}, i \equiv 2(4)$.

This is really a paraphrase of the fact that two elements in $Q_{i}$, $i \geqq 5$, are equal if and only if they have the same index-Arf invariant. 
Recent work of $\mathrm{F}$. Quinn suggests that this is true for $i=3,4$ as well. We conjecture that this is true, in fact, for all $i$. (For the definition of the Arf invariant on $\Omega_{4 i+2}^{S}$, see [1].)

3. Now let $N$ be a compact PL manifold of dimension $r$, and let $\xi: E \rightarrow B$ be a $(k-1)$-spherical fibration $T(\xi)=\mathfrak{N}_{\xi} \cup c E$. Let $f: N$ $\rightarrow T(\xi)$. Set $R=f^{-1}\left(\mathfrak{N}_{\xi}\right), Q=f^{-1}(c E), D=f^{-1}(E), \quad\left(E=\Re_{\xi} \cap c E\right)$ so that $D=R \cap Q$. We say that $f$ is transverse regular iff $R, Q$ are codimension 0 submanifolds of $N, \partial R=\partial Q=D$ and

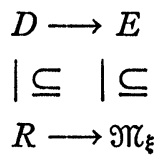

is, up to homotopy, a map of $(k-1)$-spherical fibrations. Thus, $R$ is a P.D. space of dimension $r-k$. A similar definition can be made if $N$ is a manifold with boundary.

THEOREM 1. Let $N$, $\xi$ be as above, with $k \geqq 6, r \leqq 3 k-3$, or with $k \geqq 3, r \leqq 2 k-1$. Let $f: N \rightarrow T(\xi)$ be a continuous map. Then there are obstructions to deforming $f$ to a transverse regular map which lie in $H^{i}\left(N, Q_{i-k-1}\right)$.

Actually, for a suitable definition of transverse regularity, Theorem 1 holds true for $N$ an arbitrary complex.

Theorem 1 can be elucidated by considering the following construction:

First, $T(\xi)-*$ is an open set $a$ of $T(\xi)$. (Here, $*$ is the cone point of $c E$.) We may assume that there is a contractible open set $O \subseteq B$; $\mathfrak{T}_{\xi \mid \mathcal{E}} \subseteq \mathfrak{T}_{\xi}$, and $\mathfrak{T}_{\xi \mid \mathcal{O}} \cup(c E-E)$ is an open set $B$ of $T(\xi) .\{Q, B\}$ is an open cover of $T(\xi)$. Let $\bar{\omega}(\xi)$ be the semisimplicial complex whose $j$ simplices are maps $\sigma: \Delta^{i} \rightarrow T(\xi)$ where $\sigma\left(\Delta^{i}\right) \subseteq Q$ or $\sigma\left(\Delta^{j}\right) \subseteq ß$ and where $\sigma$ is transverse regular on $\Delta^{i}$ and all its faces. There is a natural map $\bar{\omega}(\xi) \rightarrow T(\xi)$. The obstructions of Theorem 1 measure the possibility that a map to $T(\xi)$ may be deformed so that it can be lifted to $\bar{\omega}(\xi)$. If $T$ is a Generalized Thom Spectrum defined by $\xi_{k}, k=1,2, \cdots$ then we can make $\left\{\bar{\omega}\left(\xi_{k}\right)\right\}$ into a spectrum $\overline{\mathscr{W}}(T)$ so that the maps $\bar{\omega}\left(\xi_{k}\right)$ $\rightarrow T\left(\xi_{k}\right)$ define a map of spectra $\bar{W}(T) \rightarrow T$.

We can define a semisimplicial complex $\omega(\xi)$ similar to $\bar{\omega}(\xi)$ by simply ignoring the condition that image $\sigma \subseteq Q$ or $B$, and requiring only that $\sigma$ be transverse regular on $\Delta^{j}$ and all its faces. Thus $\bar{\omega}(\xi)$ $\subseteq \omega(\xi)$. Similarly, given a Generalized Thom Spectrum, the complexes $\omega\left(\xi_{k}\right)$ form a spectrum $w(T)$. It turns out that $\pi_{*}{ }^{\top} W(T)=\Omega_{*}^{T}$, and that the natural map $\mathscr{W}(T) \rightarrow T$ is a representative, on the level of spectra, 
of the Pontrjagin-Thom map.

THEOREM 2. There is an exact sequence

$$
\cdots \rightarrow Q_{i} \rightarrow \pi_{i} \bar{W}(T) \rightarrow \pi_{i}(T) \rightarrow Q_{i-1} \rightarrow \cdots .
$$

This follows from Theorem 1.

We conjecture that $\overline{\mathscr{W}}(T) \subseteq \mathscr{W}(T)$ is a homotopy equivalence. This would allow the substitution of $\Omega_{i}^{T}$ for $\pi_{i} \bar{W}(T)$ in the exact sequence above.

4. Consider a Generalized Thom Spectrum $T$. If $(M, f)$ is a $T$-space and if $g: M^{\prime} \rightarrow M$ is a degree-one map covered by $\psi_{k}: \nu_{k}\left(M^{\prime}\right) \rightarrow \nu_{k}(M)$, large $k$, then setting $f_{k}^{\prime}=f_{k} \circ \psi_{k}$ defines another $T$-space $\left(M^{\prime}, f^{\prime}\right)$. We say that $\left(M^{\prime}, f^{\prime}\right)$ is related to $\left(M^{\prime}, f^{\prime}\right)$. Consider the equivalence relation $\cong$ generated by this relation; dividing by $\cong$ defines a quotient group $T_{*}^{T}$ of $\Omega_{*}^{T}$, and it is easy to see that $p: \Omega_{*}^{T} \rightarrow \pi_{*} T$ factors through $p_{0}: T_{*}^{T} \rightarrow \pi_{*} T$.

THEOREM 3. $p_{0}$ is a monomorphism; in fact, for $i \geqq 6, \neq 4 k+3, p_{0}$ is an isomorphism; for $i \geqq 7,=4 k+3$, coker $p_{0}$ is at most $Z_{2}$.

Theorem 3 follows from Theorem 1 and some of the techniques of Theorem 2. We outline the proof: To show $p_{0}$ is monic, we first show that if $M, f$ represents an element in the kernel of $p_{0}: T_{i}^{T} \rightarrow \pi_{i} T$ then there is an $M^{1}, f^{1}$ related to $M, f$ so that $\left[M^{1}, f^{1}\right] \in \Omega_{i}^{T}$ is in the image of $\pi_{i} \bar{W}(T) \rightarrow \pi_{i}{ }^{\top} \mathcal{W}(T)=\Omega_{i}^{T}$. One then shows by means of the exact sequence that $M^{1}, f^{1}$ is $T$ cobordant to $M^{2}, f^{2}$ so that $M^{2}, f^{2}$ is actually a $G$-framed P.D., space, i.e. $M^{2}, f^{2}$ is an $S$-space, and represents an element in $Q_{i}$. It is easy to show then that there is a $T$-space (in fact, an $S$-space) $M^{3}, f^{3}$ related to $M^{2}, f^{2}$, and $M^{3}, f^{3}$ is $T$-cobordant (in fact, $S$-cobordant) to zero. Thus $M, f$ represents zero in $T_{i}^{T}$, and $p_{0}$ is monic.

To show the rest of Theorem 3, we merely use the computation of $Q_{*}$ of Lemma 1.

5. Some conjectures are suggested by the above. First, let $O^{i}$ denote $i$ th loop space (instead of the customary notation $\Omega^{i}$ ). Then given the spectrum $T, \mathscr{W}(T)$, we get spaces $A(T)=\lim O^{i} T\left(\xi_{i}\right)$, $B(T)=\lim O^{i} \omega\left(\xi_{i}\right)$ so that $\pi_{*} A(T)=\pi_{*} T, \pi_{*} B(T)=\pi_{*}{ }^{W}(T)=\Omega_{*}^{T}$. The map of spectra $W(T) \rightarrow T$ becomes a map $B(T) \rightarrow A(T)$; and if we have a map of Generalized Thom Spectra $T \rightarrow U$ we get a homotopy commutative square

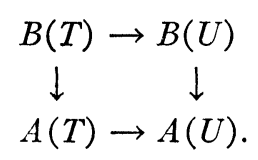


Let $C(T)=$ fiber $B(T) \rightarrow A(T)$. Then we conjecture that $C(T)=G /$ Top and

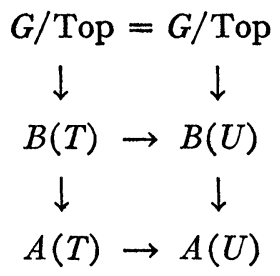

is a map of fibrations. If we abandon the condition that $T$ be defined by orientable fibrations $S_{k}$, we then conjecture that $C(T)$ is a space whose homotopy realizes the $L$-groups of some appropriate group (see [3], [4]).

\section{BIBLIOGRAPHY}

1. W. Browder, The Kervaire invariant of framed manifolds and its generalization, Ann. of Math. (2) 90 (1969), 157-186.

2. - Surgery on simply connected manifolds (to appear).

3. F. Quinn, Thesis, Princeton University, Princeton, N. J., 1969.

4. C. T. C. Wall, Surgery on non-simply-connected manifolds, Ann. of Math. (2) 84 (1966), 217-276. MR 35 \#3692.

Courant Institute of Mathematical Sciences New York University, New YORK, NEW YORK 10012

Rutgers University-The State University of New Jersey, New BrunsWICK, New JeRsey 08903 